\title{
BACTERIAS ÁCIDO LÁCTICAS PRODUCTORAS DE RIBOFLAVINA AISLADAS DEL PROCESO DE ELABORACIÓN DE LA "TUNTA"
}

\section{PRODUCTION OF RIBOFLAVIN BY LACTIC ACID BACTERIA ISOLATED FROM THE MANUFACTURING OF "TUNTA"}

\author{
Ricardo Santos-Mendoza ${ }^{1}$, Elena Ramos-Vásquez ${ }^{1}$, \\ Amparo Iris Zavaleta ${ }^{2}$, Doris Zúñiga-Dávila ${ }^{1}$ y Carmen Velezmoro-Sánchez ${ }^{3}$
}

\begin{abstract}
Resumen
En el presente estudio, se seleccionaron e identificaron bacterias de ácido láctico (BAL) productoras de riboflavina, aisladas durante el proceso de elaboración de Tunta, una patata nativa peruana con un proceso tradicional de congelación y deshidratación para el consumo y conservación. De las 109 cepas BAL aisladas, 32 fueron las que presentaron producción de riboflavina y sólo cuatro mostraron un nivel alto entre $334 \mathrm{ng} / \mathrm{ml}$ a $426 \mathrm{ng} / \mathrm{ml}$ en medio libre de riboflavina. La caracterización molecular de estas cuatro cepas mostró que están relacionados filogenéticamente con Leuconostoc mesenteroides. Otros taxones identificados tras la secuenciación del gen 16S rRNA fueron Lactobacillus curvatus, L. mesenteroides subsp. dextranicum y Lactobacillus sakei. Posteriores estudios con estas cepas BAL productoras de riboflavina permitirán revalorizar los nutrientes de la Tunta como alimento nativo del Perú.
\end{abstract}

Palabras clave: bacterias ácido lácticas (BAL), gen ribosómico 16s Rrna, riboflavina, tunta, Jallamilla.

\begin{abstract}
In the present study, we selected and identified riboflavin-producing lactic acid bacteria (LAB), isolated 'during the process of manufacturing Tunta, one Peruvian native potato with traditional process of dehydration and freezing for comsumption and conservation. From 109 LAB isolates obtained, 32 were capable to produce riboflavin and only four showed a high level of the vitamin between $334 \mathrm{ng} / \mathrm{ml}$ and $426 \mathrm{ng} / \mathrm{ml}$ in riboflavin-free medium. The molecular characterization of the four strains allowed their identification with a $95 \%$ of similarity as Leuconostoc mesenteroides. Other strains were identified on the basis of 16S rRNA sequencing, as Lactobacillus curvatus, $L$. mesenteroides subsp. dextranicum and Lactobacillus sakei. Further studies with these riboflavinproducing LAB strains will allow to give value to Tunta's nutrients as native Peruvian food.
\end{abstract}

Key words: lactic acid bacteria (LAB), ribosomal gen $16 \mathrm{~S}$ rRNA, riboflavin, tunta, Jallamilla.

\section{Introducción.}

En el mundo se producen más de 3500 tipos de alimentos fermentados (Bibek, 2004). Perú también produce alimentos nativos fermentados de origen nativo provenientes de diversos granos y tubérculos. La Tunta es un producto fermentado a partir de papas nativas amargas, producidas a $4000 \mathrm{msnm}$ mediante procesos de inmersión en agua, congelado y secado natural. Durante la etapa de inmersión de las papas en el agua de río, se han determinado poblaciones microbianas de bacterias ácido lácticas (BAL) en altas concentraciones (Ramos et al., 2018). Las BAL y levaduras han sido identificadas como los microorganismos predominantes involucrados en el proceso de fermentación de productos alimenticios, y que a veces actúan en un consorcio microbiano complejo (Blandino et al., 2003).
Las BAL sintetizan un largo espectro de moléculas con propiedades promotoras de la salud tales como ácido aminobutírico (GABA), feniletilamina, péptidos bioactivos, ácidos grasos de cadena corta, ácido linoleico, selenometabolitos y vitaminas (Pessione, 2012). Las vitaminas, en especial las del complejo B, son producidas industrialmente por síntesis química a partir de ribosa (Kurth et al., 1996). Además, las BAL son usadas en la síntesis de estas vitaminas para alimentos funcionales (Capozzi et al., 2011; Samaniego-Vaesken et al., 2012) y con posibilidad de reducir riesgo de enfermedades especificas crónicas (Mazzoli et al., 2014).

Las vitaminas del complejo B producidas por bacterias acido lácticas han sido empleadas en la elaboración de productos alimenticios enriquecidos. Así, Guru \& Viswanathan (2013) consiguieron una producción de riboflavina $2 \quad 930 \quad \mu \mathrm{g} / 1$ usando 
Tabla 1. Identificación molecular de BAL productoras de riboflavina aisladas de la cadena de elaboración de Tunta.

\begin{tabular}{clc}
\hline Código cepa & Identificación molecular (según secuencia del & $\begin{array}{c}\text { Riboflavina } \\
\text { (ng/ml) }\end{array}$ \\
\hline 2TP02-BAL23 & Lactobacillus curvatus & $84 \pm 7$ \\
2TP06-BAL18 & Lactobacillus curvatus & $112 \pm 4$ \\
2TP02-BAL24 & Leuconostoc mesenteroides subsp. dextranicum & $21 \pm 3$ \\
2TP12-BAL03 & Lactobacillus sakei & $12 \pm 2$ \\
2TP01-BAL05 & Lactobacillus curvatus & $10 \pm 1$ \\
2TP05-BAL19 & Lactobacillus curvatus & $35 \pm 3$ \\
2TP01-BAL13 & Leuconostoc mesenteroides subsp. dextranicum & $117 \pm 12$ \\
2TP12-BAL04 & Lactobacillus sakei & $79 \pm 2$ \\
2TP03-BAL11 & Leuconostoc mesenteroides & $57 \pm 2$ \\
2TP10-BAL18 & Leuconostoc mesenteroides & $377 \pm 12$ \\
2TP08-BAL04 & Leuconostoc mesenteroides & $420 \pm 15$ \\
2TP08-BAL09 & Leuconostoc mesenteroides & $334 \pm 10$ \\
2TP01-BAL04 & Leuconostoc mesenteroides subsp. dextranicum & $171 \pm 3.1$ \\
2TP02-BAL01 & Leuconostoc mesenteroides & $426 \pm 12$ \\
2TP02-BAL13 & Lactobacillus curvatus & $19 \pm 1$ \\
2TP03-BAL17 & Leuconostoc mesenteroides subsp. dextranicum & $20 \pm 2$ \\
2TP01-BAL03 & Leuconostoc mesenteroides & $36 \pm 4$ \\
2TP02-BAL15 & Leuconostoc mesenteroides subsp. dextranicum & $62 \pm 2$ \\
2TP10-BAL20 & Leuconostoc mesenteroides & $12 \pm 2$ \\
2TP06-BAL10 & Leuconostoc mesenteroides & $57 \pm 3$ \\
2TP02-BAL04 & Leuconostoc mesenteroides & $67 \pm 7$ \\
2TP10-BAL21 & Lactobacillus curvatus & $142 \pm 21$ \\
2TP06-BAL11 & Lactobacillus curvatus & $16 \pm 3$ \\
2TP06-BAL30 & Lactobacillus curvatus & $4 \pm 1$ \\
2TP08-BAL08 & Lactobacillus curvatus & $89 \pm 5$ \\
2TP05-BAL08 & Leuconostoc mesenteroides & $41 \pm 3$ \\
2TP02-BAL14 & Lactobacillus sakei & $29 \pm 1$ \\
2TP08-BAL10 & Lactobacillus curvatus & $56 \pm 7$ \\
2TP03-BAL14 & Lactobacillus curvatus & $165 \pm 5$ \\
2TP02-BAL12 & Lactobacillus sakei & $75 \pm 3$ \\
2TP10-BAL06 & Lactobacillus curvatus & $130 \pm 10$ \\
2TP10-BAL05 & Leuconostoc mesenteroides & $64 \pm 2$ \\
\hline & &
\end{tabular}

probióticos como Lactobacillus acidophilus y Lactococcus lactis aislados de cuajada y queso. De igual forma, Juarez del Valle et al. (2014), reportaron estudios de la cepa Lactobacillus plantarum CRL 725 con capacidad de incrementar significativamente una concentración inicial de riboflavina en leche de soja de $309 \pm 9 \mathrm{ng} / \mathrm{ml}$ a $700 \pm 20 \mathrm{ng} / \mathrm{ml}$ después de 12 horas de incubación a $37^{\circ} \mathrm{C}$.

El objetivo del presente trabajo fue seleccionar y caracterizar molecularmente cepas BAL productoras de riboflavina $\left(\mathrm{B}_{2}\right)$ aisladas de papas amargas sometidas a un proceso de fermentación natural llamado Tunta.

\section{Materiales y métodos.}

Reactivación de cepas BAL aisladas de Tunta.

Para la selección de cepas BAL con capacidad de producir riboflavina, se reactivaron 109 cepas BAL del Banco de Cepas del Laboratorio de Ecología Microbiana y Biotecnología, que fueron aisladas de diferentes etapas de la cadena de elaboración de la Tunta, actividad realizada en la comunidad de Jallamilla en la provincia de El Collao, Ilave (Puno, Perú) a $3860 \mathrm{msnm}$. Todas ellas fueron incubadas en caldo MRS (De Manet at., 1960) a $28^{\circ} \mathrm{C}$ sin agitación.
Selección de cepas BAL productoras de Riboflavina.

Luego de 24 horas de crecimiento de las cepas en caldo MRS, se tomó una alícuota de $1 \mathrm{ml}$ y se centrifugó a $8000 \mathrm{rpm}$ por 5 minutos. El pellet se resuspendió con $1 \mathrm{ml}$ de solución $\mathrm{NaCl} 0.85 \%$ estéril y se centrifugó a $8000 \mathrm{rpm}$ por 5 minutos. Esto se repitió dos veces para obtener un lavado de las células. Las células resuspendidas fueron inoculadas $(4 \% \mathrm{v} / \mathrm{v})$ en un tubo con medio libre de riboflavina (MLR) y se incubó a $28{ }^{\circ} \mathrm{C}$ durante $24-48 \mathrm{~h}$ en oscuridad. Fue repetido este procedimiento cuatro veces, y sólo los que alcanzaron un buen crecimiento en este último paso, fueron seleccionados como BAL productoras de riboflavina. Cuando la cepa no evidenció crecimiento, se inoculó nuevamente en el MLR ( $4 \%$ v/v) y $\operatorname{MLR}+B_{2}(0.1 \%$ a 1 $\mathrm{mg} / \mathrm{ml})$, incubando a $28{ }^{\circ} \mathrm{C}$ durante $16 \mathrm{~h}$ en oscuridad, para determinar si la cepa se adecua o no al medio.

Para extraer la riboflavina producida por la cepa, se extrajo la muestra total. Se mezclaron $500 \mu \mathrm{l}$ de muestra con $500 \mu 1$ de ácido acético al 1\%. Se incubó a $100{ }^{\circ} \mathrm{C}$ durante 5 minutos y luego se centrifugó a 5000 g durante 5 minutos y se separó el sobrenadante que contenía la vitamina. Todas las muestras se conservaron a $-20{ }^{\circ} \mathrm{C}$. 
Cuantificación de vitamina por método de espectrofotómetro de microplacas.

Se reactivó la cepa de referencia Lactobacillus rhamnosus ATCC7469 en el medio MRS incubado a $37{ }^{\circ} \mathrm{C}$ durante 16-24 horas. Se lavó dos veces con solución $\mathrm{NaCl} 0.85 \%$ y se inoculó al $4 \%$ en MLR. Se colocaron $100 \mu \mathrm{l}$ de la cepa de referencia en cada pocillo de la microplaca y $100 \mu \mathrm{l}$ de las muestras y las concentraciones estándar de riboflavina $(0,10,25,50$, 75, 100, 150, 200, 400 ng/ml). Finalmente, se incubó a $37{ }^{\circ} \mathrm{C}$ durante 48 horas en oscuridad y la lectura de absorbancia de las microplacas fue a $600 \mathrm{~nm}$ (Golbach et al., 2007).

Identificación Molecular de BAL aisladas de Tunta.

El gen ribosomal $16 \mathrm{~S}$ rRNA fue amplificado por PCR usando los primers fD1 (5'CCGAATTCGTCGACAACAGAGTTTGATCCTGG CTCAG-3') y CCCGGGATCCAAGCTTAAGGAGGTGATCCAG

CC-3') como se describe en Versalovic et al., (1991). El ADN fue amplificado en $25 \mu 1$ de volumen en una mezcla de BOX-PCR conteniendo $5 \mu \mathrm{l}$ de ADN, 0.2 $\mathrm{mM}$ dNTPs, $1.5 \mathrm{mM} \mathrm{MgCl}_{2}, 0.2 \mathrm{pmol} / \mathrm{ml}$ de cada primer, $0.02 \mathrm{U} / \mu \mathrm{l}$ Taq DNA polymerasa (ThermoScientific, USA) y $1 \times$ buffer $\mathrm{KCl}$ (Versalovic et al., 1991). El ADN fue amplificado bajo las siguientes condiciones: desnaturalización inicial a 93 ${ }^{\circ} \mathrm{C}$ por dos minutos, 30 ciclos de $93{ }^{\circ} \mathrm{C}$ por $45 \mathrm{~s}, 62{ }^{\circ} \mathrm{C}$ por $45 \mathrm{~s}$ (alineación), y $72{ }^{\circ} \mathrm{C}$ por $2 \mathrm{~min}$ (extensión), con una final extensión a $72{ }^{\circ} \mathrm{C}$ por $5 \mathrm{~min}$. El producto de PCR fue purificado con un kit PCR Cleanup Kit (ThermoScientific, USA) de acuerdo a como menciona el protocolo y posteriormente enviado a ser secuenciado a Macrogen Inc. (Seúl, Corea del Sur). Para la limpieza de secuencia se usaron los programas BioEdit 2.0 juntamente con el BLAST (blast.ncbi.nlm.nih.gov) y la identificación con la base de datos EZTAXON (Kimet al., 2012). El árbol filogenético fue realizado con los programas Clustal X.2.0.5 y Mega 5.05.

\section{Resultados y discusión.}

Selección de bacterias productoras de riboflavina.

El uso de BAL capaces de producir nutraceúticos en alimentos ofrece muchas ventajas sobre el de síntesis química, ya que es más barato, sus fuentes son renovables, incrementan el valor nutricional de alimentos y muestran una alta aceptación de consumidores por ser alimentos naturales (Juárez del Valle et al., 2014). En el ensayo de las 109 cepas BAL, 32 fueron capaces de crecer tras cuatro pases en medio de cultivo libre de riboflavina (MLR). De todas ellas, sólo 4 cepas, 2TP10-BAL18, 2TP08-BAL04, 2TP08BAL09 y 2TP02-BAL01 fueron seleccionadas por su alta productividad de riboflavina (Tabla 1). El criterio de selección fue la mayor producción de riboflavina total y de crecimiento óptimo en medio MLR.
Las cuatro cepas seleccionadas mostraron niveles desde $334 \mathrm{ng} / \mathrm{ml}$ hasta valores altos como $426 \mathrm{ng} / \mathrm{ml}$, mostrando niveles superiores de $\mathrm{B}_{2}$ a partir de $\mathrm{BAL}$ descritas por Capozzi et al. (2011) y competentes descritas por Juarez del Valle et al. (2014) y Pacheco et al. (2016). La riboflavina es una vitamina esencial para el desarrollo y metabolismo celular en humano y de acuerdo a la Organización Mundial de la Salud, la ingesta diaria es de 0.9 a $1.6 \mathrm{mg}$ (FAO/WHO, 2002). A pesar de que los resultados mostrados aún se encuentran bajos, estas cepas productoras pueden llevarse a una optimización para sobreproducción tal como lo ha mostrado en otro trabajo Juárez del Valle $e t$ al. (2014).

Caracterización Molecular de las BAL productoras de riboflavina.

De las 32 cepas BAL, 12 cepas corresponden al grupo de los Lactobacillus curvatus, 11 cepas al grupo Leuconostoc mesenteroides, 5 cepas al grupo $L$. mesenteroides subsp. dextranicum y 4 cepas al grupo $L$. sakei (Tabla 1).

Las cuatro cepas que fueron seleccionadas con mayor nivel de riboflavina (Tabla 1) están relacionadas filogenéticamente a $L$. mesenteroides (Figura 1), y pueden ser usadas en la industria alimentaria, al igual que las especies $L$. plantarum, $L$. lactis, $L$. mesenteroides y Propionibacterium freudenreichii mencionadas por Burgess et al. (2006).

\section{Conclusiones.}

En el presente estudio se seleccionaron las bacterias ácido lácticas, 2TP10-BAL18, 2TP08-BAL04, 2TP08BAL09 y 2TP02- BAL01 con niveles de producción de riboflavina hasta $426 \mathrm{ng} / \mathrm{ml}$, aisladas del proceso de elaboración de Tunta, y las cuatro cepas están relacionadas filogenéticamente con $L$. mesenteroides. Las BAL productoras de riboflavina permitirán revalorizar la microbiota de la Tunta, alimento nativo del Perú. Así también se podrá optimizar la sobreproducción de esta vitamina o usar las cepas como starters en la fermentación de papas amargas u otros alimentos.

\section{Agradecimientos.}

Al grupo Consorcio "Los Aymaras" por las facilidades en la recolección de las muestras de Tunta, así también al financiamiento del Programa Nacional de Innovación para la Competitividad y Productividad, Innovate Perú Contrato $\mathrm{N}^{\circ}$ 223-FINCyT-IA-2013 Lima, Perú. 


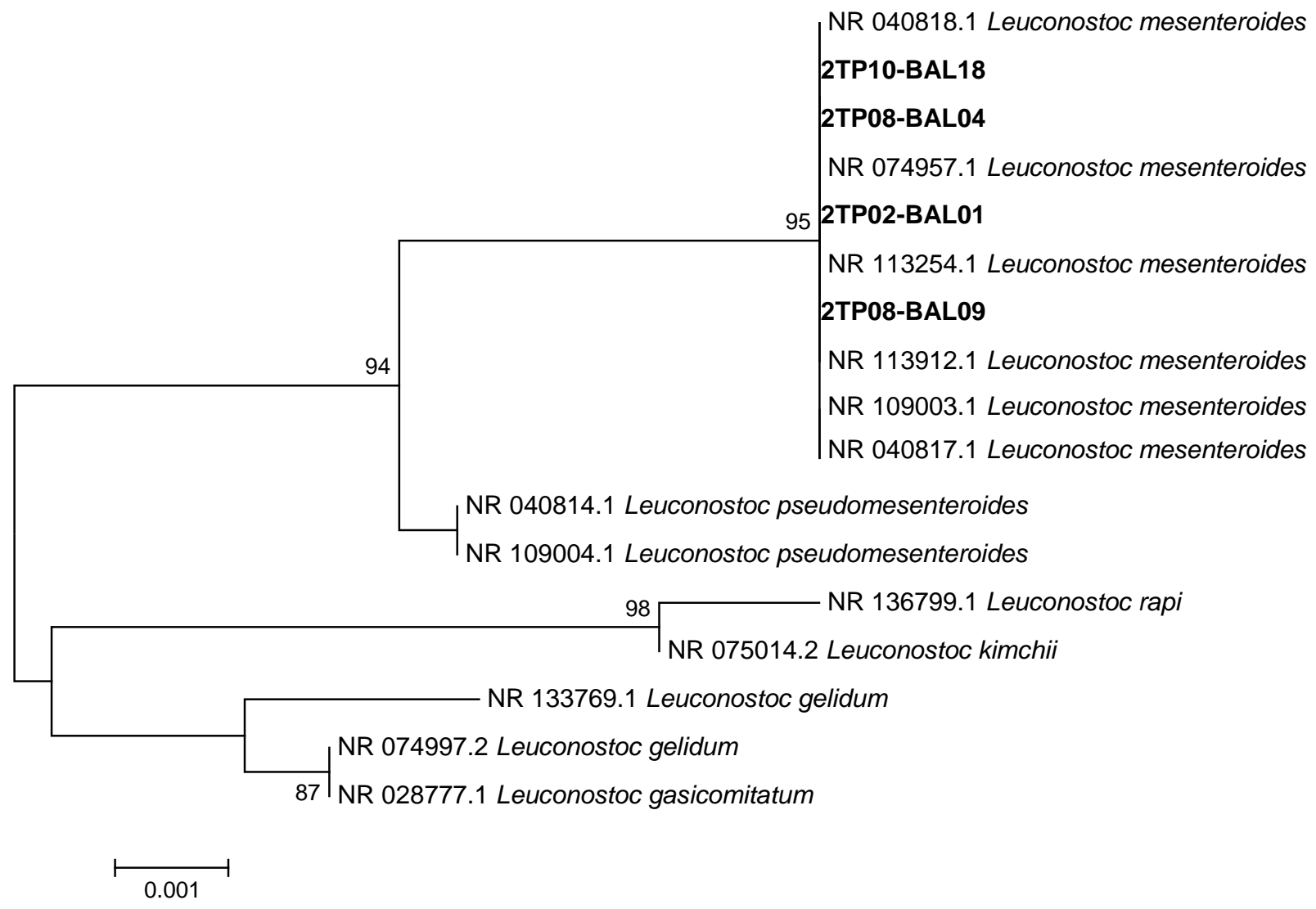

Figura 1. Árbol Filogenético con la prueba Neighbor-Joining de las bacterias acido lácticas con alto nivel de producción de riboflavina aisladas de la cadena de elaboración de la Tunta. (Cepas 2TP10-BAL18, 2TP08BAL-04, 2TP02-BAL01 у 2TP08-BAL09).

\section{Literatura citada.}

Bibek R. 2004. Fundamental Food Microbiology. Third Edition. CRC Press, Washington D.C.

Blandino A., Al-Aseeri M., Pandiella S., Cantero, D. \& Webb C. 2003. Cereal-based fermented foods and beverages. Food Res. Int. 36: 527-543.

Burgess C., Smid E., Rutten G. \&Van Sinderen D. 2006. A general method for selection of riboflavin-overproducing food grade microorganisms. Microbial Cell Factories. 5: 24.

Capozzi V., Menga V., Digesu A. M., De Vita P., Van Sinderen D. \& Cattivelli L. 2011. Biotechnological production of vitamin B2-enriched bread and pasta. Journal of Agricultural and Food Chemistry. 59: 80138020.

De Man J. D., Rogosa M., \& Sharpe M. E. 1960. A medium for the cultivation of lactobacilli. Journal of Applied Bacteriology. 23: 130-135.

FAO/WHO. 2002. Human vitamin and mineral requirements. Bangkok, Thailand.

Golbach J.L., Chalova V.I., Woodward C.L. \& Ricke S.C. 2007. Adaption of Lactobacillus rhamnosus riboflavin assay to microtiter plates. Journal of Food Composition and Analysis. 20: 568-574.
Guru V. \& Viswanathan K. 2013. Riboflavin production in milk whey using probiotic bacteria - Lactobacillus acidophilus and Lactococcus lactis. Indian Journal of Fundamental and Applied Life Sciences. 3(4): 169-176.

Juárez del Valle M., Laiño J.E., Savoy de Giori \& LeBlanc J.G. 2014. Riboflavin producing lactic acid bacteria as a biotechnological strategy to obtain bio-enriched soymilk. Food Research International. 62: 1015-1019.

Kim O., Cho Y., Lee K., Yoon S., Kim M., Na H., Park S., Jeon Y., Lee J., Yi H., Won S. \& Chun J. 2012. Introducing EzTaxon-e: a prokaryotic 16S rRNA Gene sequence database with phylotypes that represent uncultured species. Int. J. Syst. Evol. Microbiol . 62: 716721.

Kurth R., Paust J. \& Hanlein W. 1996. Riboflavin. Ullmann's Encyclopedia of Industrial Chemistry. Weinheim, Germany: Wiley-VCH. 521-530.

Mazzoli R., Bosco F., Mizrahi I., Bayer E. \& Pessione E. 2014. Towards lactic acid bacteria-based biorefineries. Biotechnology Advances 32: 1216-1236.

Pacheco F., Biscola V., Le Blanc J. \& Gombossy B. 2016. Effect of indigenous lactic acid bacteria isolated from goat milk and cheeses on folate and riboflavin content of fermented goat milk. LWT - Food Science and Technology. 71: 155-161. 
Pessione E. 2012. Lactic acid bacteria contribution to gut microbiota complexity: lights and shadows. Front Cell Infect Microbiol. 2:86.

Ramos E., Santos R., Velásquez E., Velezmoro C. \& Zúñiga D. 2018. Genetic Diversity and antimicrobial activity of lactic acid bacteria in the preparation of traditional fermented potato product "tunta". World Journal of Microbiology and Biotechnology. 34: 144.
Samaniego-Vaesken M., Alonso-Aperte E. \& VarelaMoreiras G. 2012. Vitamin food fortification today. Food \& Nutrition Research. 56: 5459.

Versalovic J., Koeuth T. \& Lupski J.R. 1991. Distribution of repetitive DNA sequences in eubacteria and application to fingerprinting of bacterial genomes. Nucl. Acids Res. 19: 6823-6831.

1 Laboratorio de Ecología Microbiana y Biotecnología, Departamento de Biología-Facultad de Ciencias, Universidad Nacional Agraria La Molina. Av. La Molina s/n. Lima 12, Perú. rsantosme@gmail.com.

${ }^{2}$ Laboratorio de Biología Molecular. Facultad de Farmacia y Bioquímica. Universidad Nacional Mayor de San Marcos. Jr. Puno N¹002. Lima 1, Perú.

${ }^{3}$ Departamento de Ingeniería de Alimentos. Facultad de Industrias Alimentarias, Universidad Nacional Agraria La Molina. 